\title{
Genome-wide evolutionary characterization and analysis of bZIP transcription factors and their expression profiles in response to multiple abiotic stresses in Brachypodium distachyon
}

Xiang Liu and Zhaoqing Chu*

\begin{abstract}
Background: Plant basic leucine zipper (bZIP) transcription factors are one of the largest and most diverse gene families and play key roles in regulating diverse stress processes. Brachypodium distachyon is emerging as a widely recognized model plant for the temperate grass family and the herbaceous energy crops, however there is no comprehensive analysis of bZIPs in B. distachyon, especially those involved in stress tolerances.

Results: In this study, 96 bZIP genes (BdbZIPs) were identified distributing unevenly on each chromosome of $B$. distachyon, and most of them were scattered in the low CpG content regions. Gene duplications were widespread throughout $B$. distachyon genome. Evolutionary comparisons suggested B. distachyon and rice's bZIPs had the similar evolutionary patterns. The exon splicing in BdbZIP motifs were more complex and diverse than those in other plant species. We further revealed the potential close relationships between BdbZIP gene expressions and items including gene structure, exon splicing pattern and dimerization features. In addition, multiple stresses expression profile demonstrated that BdbZIPs exhibited significant expression patterns responding to 14 stresses, and those responding to heavy metal treatments showed opposite expression pattern comparing to the treatments of environmental factors and phytohormones. We also screened certain up- and down-regulated BdbZIP genes with fold changes $\geq 2$, which were more sensitive to abiotic stress conditions.

Conclusions: BdbZIP genes behaved diverse functional characters and showed discrepant and some regular expression patterns in response to abiotic stresses. Comprehensive analysis indicated these BdbZIPs' expressions were associated not only with gene structure, exon splicing pattern and dimerization feature, but also with abiotic stress treatments. It is possible that our findings are crucial for revealing the potentialities of utilizing these candidate BdbZIPs to improve productivity of grass plants and cereal crops.
\end{abstract}

Keywords: Abiotic stresses, Basic leucine zipper (bZIP), Brachypodium distachyon, Evolutionary comparisons, Gene expression, Heavy metals

\footnotetext{
* Correspondence: zqchu@sibs.ac.cn

Shanghai Chenshan Plant Science Research Center, Shanghai Chenshan Botanical Garden, Shanghai Key Laboratory of Plant Functional Genomics and Resources, Shanghai Institutes for Biological Sciences, Chinese Academy of Sciences, 3888 Chenhua Road, 201602 Shanghai, Songjiang, China
} 


\section{Background}

In plants, the leucine zipper (bZIP) transcription factors are one of the largest and most conserved gene families and play key roles in regulating diverse biological processes [1-5]. The bZIP domain contains about 60 to 80 amino acids and characteristically harbors two distinct function regions: a highly conserved basic region $\mathrm{N}-\mathrm{x} 7-$ $\mathrm{R} / \mathrm{K}-\mathrm{x} 9$ and a less conserved leucine zipper coiled-coil motif [6]. And the basic region and leucine zipper coiled-coil motif region was linked by a hinge region. The bZIP proteins bind to DNA by forming heterotypic or homotypic complexes $[7,8]$. The basic region is responsible for nuclear localization and DNA binding specifically, and the following leucine zipper motif consisting of several repeats of leucine or other hydrophobic amino acids and grant for recognition and dimerization specificity [9]. The intron patterns within the basic and the hinge region are very important for their functional evolution due to different status of exon splicing in these regions. In plant species such as rice, maize and Arabidopsis, the patterns of those motifs exhibited regular conservation and diversity $[10,11]$. The bZIP proteins are dimerized transcription factors in all eukaryotes, and the leucine zipper is responsible for the dimerization of bZIP proteins. The rules of dimerization specificity for bZIP proteins have been depicted [12-14]. Depend upon the basis of the presence of attractive or repulsive interhelical $\mathrm{g} \leftrightarrow \mathrm{e}$ electrostatic interactions and the presence of polar or charged amino acids in the a and d positions of the hydrophobic interface of the leucine zipper region, dimerization specificity of bZIP proteins in plant species such as Arabidopsis, maize and rice have been predicted $[10,11,15]$.

The evolution of genetic network complexities in flowering plants has revealed the important roles of regulatory transcription factor evolution to physiological variation among species [16,17]. As one of important transcription factor family, the plant bZIP transcription factors play pivotal roles in developmental processes and multiple stresses in response to environmental tolerance. The ancestor of green plants possessed four bZIP genes functionally involved in oxdative stress and unfolded protein responses that are bZIP-mediated processes in all eukaryotes [18]. Furthermore, bZIP genes regulate diverse biological processes such as seed development, flower maturation, pathogen defense, and light and stress signaling $[3,6]$. Fundamentally, various transcription factors had been observed to regulate the ABAresponsive gene expression $[19,20]$. The transcriptional drought, cold, and salinity stress gene expression have been concluded [21]. So far, members of bZIP transcription factors have been identified or predicted in most of plant species analyzed [22-26]. In cucumber, 64 bZIPs were observed and all of the select genes displayed down-regulated with PEG treatment [24]. In maize, ZmbZIP17 functions as an endoplasmic reticulum stress transducer and interact with ABA-responsive ciselements (ABRE) [5]. In rice, plenty of OsbZIPs displayed different expression patterns when dealed with cold or salt stresses [10]. OsbZIP71 was strongly induced in ABA-mediated drought and salt tolerance in rice [27]. OsbZIP46 expression was strongly induced by drought, heat, and $\mathrm{ABA}$, and functions as a positive regulator of $A B A$ signaling and drought stress tolerance of rice depending on its activation [28]. OsbZIP52/RISBZ5 could function as a negative regulator in cold and drought stress environments [29]. A bZIP gene $A B I 5$ played an important role in $\mathrm{ABA}$-arrested seed germination, was robustly associated with the flower transition in Arabidopsis [30]. A bZIP gene ThbZIP1 from Tamarix hispida in response to abiotic stresses had been characterized and showed to have an increased tolerance to drought and salt. Microarray analysis had been shown that many ROS scavenging genes were up-regulated by ThbZIP1 under salt stress conditions [31]. MabZIP3 was isolated from banana fruit, it was responsive to MeJA, ABA, and chilling stress [32]. As the most dangerous pollutions, heavy metals had been regarded as the new stress factors affecting the growth of plants. Foods contaminated with heavy metal tolerance profile of different native or genemodified plant species had been applied [33-39]. Though studies had shown that bZIP transcription factors played key roles when plants grew under environmental factors and phytohormones, there was few research of bZIP genes study in heavy metal stresses so far.

B. distachyon is a new emerging model plant of Poaceae family and the first species of sequenced grass subfamily Pooideae [40]. Due to its high efficiency for genetic manipulation and compact genome, $B$. distachyon has become more crucial in applied functional genomics [41]. Researches on B. distachyon are moving forward rapidly, and the research field has covered grain development and starch deposition, biotic and abiotic stress responses, and biofuel production [42-44]. Global gene expression in $B$. distachyon had revealed extensive network plasticity in response to abiotic stress [45]. Evolutionary studies on bZIP gene families had been shown that a shifting landscape of biochemical functions related to signaling and gene expression contributed to species diversity [46]. Although these studies reported were involved in various plant species, none of researches were associated with the evolution and the molecular biology of stress in detail, especially in the grain model plant $B$. distachyon. There is no investigation of bZIP transcription factors in $B$. distachyon so far. Understanding the detailed evolutionary history of BdbZIPs and their corresponding functions in stress biology is of great importance in B. distachyon. 
In this study, we identified $B d b Z I P$ genes genomewidely in B.distachyon and further investigated their chromosomal localization and evolutionary relationship with their counterparts from monocot $O$. sativa and $\operatorname{dicot} A$. thalinana. We analyzed their exons splicing of basic and hinge region of bZIP domain, which are very important for bZIP functional evolution. We also characterized dimerization pattern within the leucine zipper motif and gene structures, and obtained tissue-specific gene expression profile and genes expression profile responding to multiple stresses including environmental factors, phytohormones, and heavy metals. This study increased our understanding of BdbZIP family genes associated with stress adaptation and tolerance, which was crucial for further study to improve the productivity of grass plants and cereal crops.

\section{Methods}

\section{Plant growth condition and treatments}

The seeds of $B$. distachyon $\mathrm{Bd} 21-3$ were surface sterilized with $20 \% \mathrm{NaOCl}$ and planted on $0.6 \%$ agar containing 0.5× Murashige and Skoog and 0.3\% Sucrose. Plants were grown at $22^{\circ} \mathrm{C}$ under 16 -h-light/8-h-dark conditions and the light intensity was $120 \mu \mathrm{m} \mathrm{m}^{-2} \mathrm{~s}^{-1}$. As for stress expression analysis, 2-week-old seedlings were treated with 3 major treatment groups including group 1-environmental factors ( cold, heat, $\mathrm{H}_{2} \mathrm{O}_{2}, \mathrm{PEG}$, and $\mathrm{NaCl})$, group 2-heavy metals $(\mathrm{Cu}, \mathrm{Zn}, \mathrm{Mn}, \mathrm{Cd}$, and $\mathrm{Pb})$ and group 3-phytohormones (SA, 6-BA, ABA, and MeJA) (As for detailed treatments, please refer to Additional file 1: Table S1).

\section{bZIP sequence extraction and structure analysis}

The sequences of $B$. distachyon bZIP genes were obtained from BGD (Brachypodium Genome Database) (http://Brachypodium.org) and Plant Transcription Factor Database verition 3.0 (Plant-TFDB 3.0) (http://planttfdb. cbi.pku.edu.cn) [47]. The Arabidopsis and rice bZIP genes were retrieved from The Arabidopsis Information Resource (TAIR), Plant-TFDB and National Rice Gene Database (http://www.ricedata.cn/gene). The status of intron and exon were annotated according to the database. All bZIP domains were verified by SMART (http://smart.embl-heidelberg.de) and Pfam (http:// pfam.sanger.ac.uk). All BdbZIP motifs were analyzed by MEME (http://meme.nbcr.net/meme). The limits of minimum width, maximum width and maximum number of motifs were specified as 10,50 and 50 respectively. Fifteen motifs including bZIP domain were finally verified with the low E-value $(<-43)$. The motifs were numbered according to their order displayed in MEME.

\section{Evolutionary analysis}

Phylogenetic and molecular evolutionary analyses were calculated in MEGA 5.0 package by using the $p$-distance model, and the Neighbor-joining statistical method followed by 1000 bootstrap replications were applied [48]. The bZIP protein sequences of $B$. distachyon, Arabidopsis and rice were loaded into MEGA 5.0. In addition, homology searches were performed in rice and Arabidopsis with BLAST, and the SCORE value and E-value were taken into account for judging the homologous genes. bZIP gene duplication events among $B$. distachyon, Arabidopsis and rice were analyzed through Plant Genome Duplication Database (http://chibba. agtec.uga.edu/duplication) [49]. The $\mathrm{Ka}$ and Ks values were listed as Additional file 1: Table S2. The data of the phylogenetic tree was deposited in Treebase Web (Accession URL: http://purl.org/phylo/treebase/phylows/ study/TB2:S17110).

\section{Chromosomal distribution and duplication of BdbZIPs}

The location information of each BdbZIP gene on each chromosome was detected from BGD (Brachypodium Genome Database). The genetic linkage map was constructed with MapDraw [50]. The number of $\mathrm{CpG}$ in every $100 \mathrm{~kb}$ scale was measured and the status of $\mathrm{CpG}$ content was constructed by PermutMatrix. Every duplicated BdbZIP gene pairs were put in same bracket.

\section{Expression data analyses}

To analyze tissue (root, stem, leaf and early spikelet) specific expression, 2-week-old seedlings and early spikelets from 5-week-old of $B$. distachyon were sampled. Total RNA was extracted as described previously [51]. The cDNA was synthesized with $3.0 \mu \mathrm{g}$ of total RNA by using PrimeScript Reverse Transcriptase (TAKARA). The reaction mixtures were diluted 20 times with distilled water and used as templates for quantitative real-time PCR. The primers used in this paper were listed in Additional file 1: Table S3. The qPCR was conducted and repeated three times. The reaction condition was as follows: $95^{\circ} \mathrm{C}$ for 3 mins, $40 \mathrm{cy}-$ cles of $95^{\circ} \mathrm{C}$ for $10 \mathrm{~s}, 55^{\circ} \mathrm{C}$ for $30 \mathrm{~s}$. The expression profiles were calculated with $-\Delta \Delta C \mathrm{~T}$ values. Fold changes were also calculated with the formula "fold change $=2^{-\Delta \Delta C T}$ ". Expression data and hierarchical clustering analysis of all the samples were carried out using PermutMatrix 1.9.3, and shown with green-red gradient. The up-regulated genes were defined as a fold change of $\geq 2$ with $p$-value $<0.05$ and marked with red color, and a fold change of $\leq 0.5$ were defined as downregulated genes with $p$-value $<0.05$ and marked with green color. All qPCR data were submitted to NCBI GEO dataset. The accession number is GSE66458. 


\section{Results and discussion} Identification of bZIP genes in plants

To investigate the evolution of bZIP genes in plant species, 21 plants from low to high grade were selected and the numbers of their bZIP genes were identified. The green plants were covered from Chlorophyta to Embyophyta subkingdoms. The evolutionary tree for these species was constructed as in Figure 1. After searching BGD and TFDB database and further validated by SMART and Pfam domain analysis, 96 unique bZIP genes were identified from $B$. distachyon. The number of bZIP genes in plants examined varied from 7 to 136 . Some plant species maintained the high number of bZIP genes, which might contribute to their numerous tandem duplications and large-scale segmental duplications [52]. Except Zea mays had a little bit more (126). bZIP genes, the plant species sub-grouped in monocot clades had approximately the close numbers (95-104), which might due that the species in this clade shared the common ancestor of Poaceae and had similar whole genome duplication [53]. B. distachyon fell into monocot and shared the same clade with Triticum aestivum, which was consistent with the notion that these two species had closer relationships. Comparing to Embrophyte, the Chlorophyta species had much less bZIPs. We inferred that high grade plants had stronger environment adaptation abilities with more bZIP genes. Speculatively, during evolution, B. distachyon and other monocot species maintained the high number of bZIP genes might contribute to large-scale segmental duplications.

\section{Chromosomal location and duplication of BdbZIP genes}

Although the basic leucine zipper transcription factors are widely distributed in all plant kingdoms, the chromosomal location of bZIP genes in B. distachyon is still unclear. Based on our analysis, bZIP genes existed in $B$. distachyon were designated as BdbZIP1-96 according to their top-to-bottom position on chromosomes from I to V (Figure 2 and Additional file 1: Table S4). BdbZIP genes were scattered on each chromosome, but their distributions were obviously not uniform in density. Certain chromosome regions had relatively high density of BdbZIP genes. Except for the smallest chromosome $\mathrm{V}$, there were one or two BdbZIP clusters on each chromosome.

To probe the potential evolutionary mechanisms of BdbZIP gene family, according to described in [50] both tandem and segmental duplication events in terms of intragenome were examined in $B$. distachyon. It was observed that gene duplications were widespread throughout $B$. distachyon lineages (Figure 2). About 66\% of $B d b Z I P$ genes were found to be duplicated at a maximal length of $100 \mathrm{~kb}$. All the duplicated genes were confined to chromosomal block duplication and none of BdbZIP genes were found to be arranged in tandem form. The phenomena were very similar to the bZIP gene family in

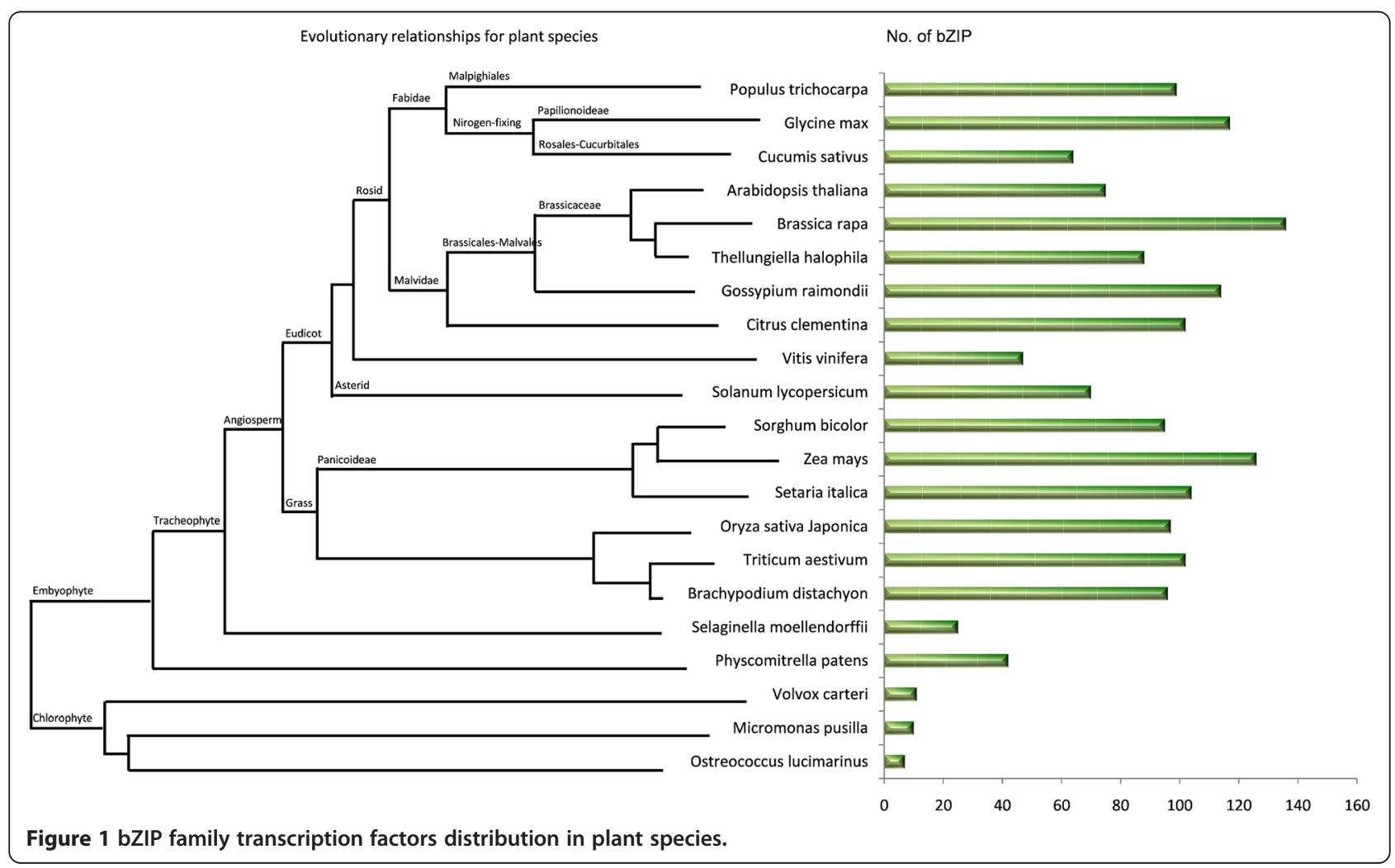




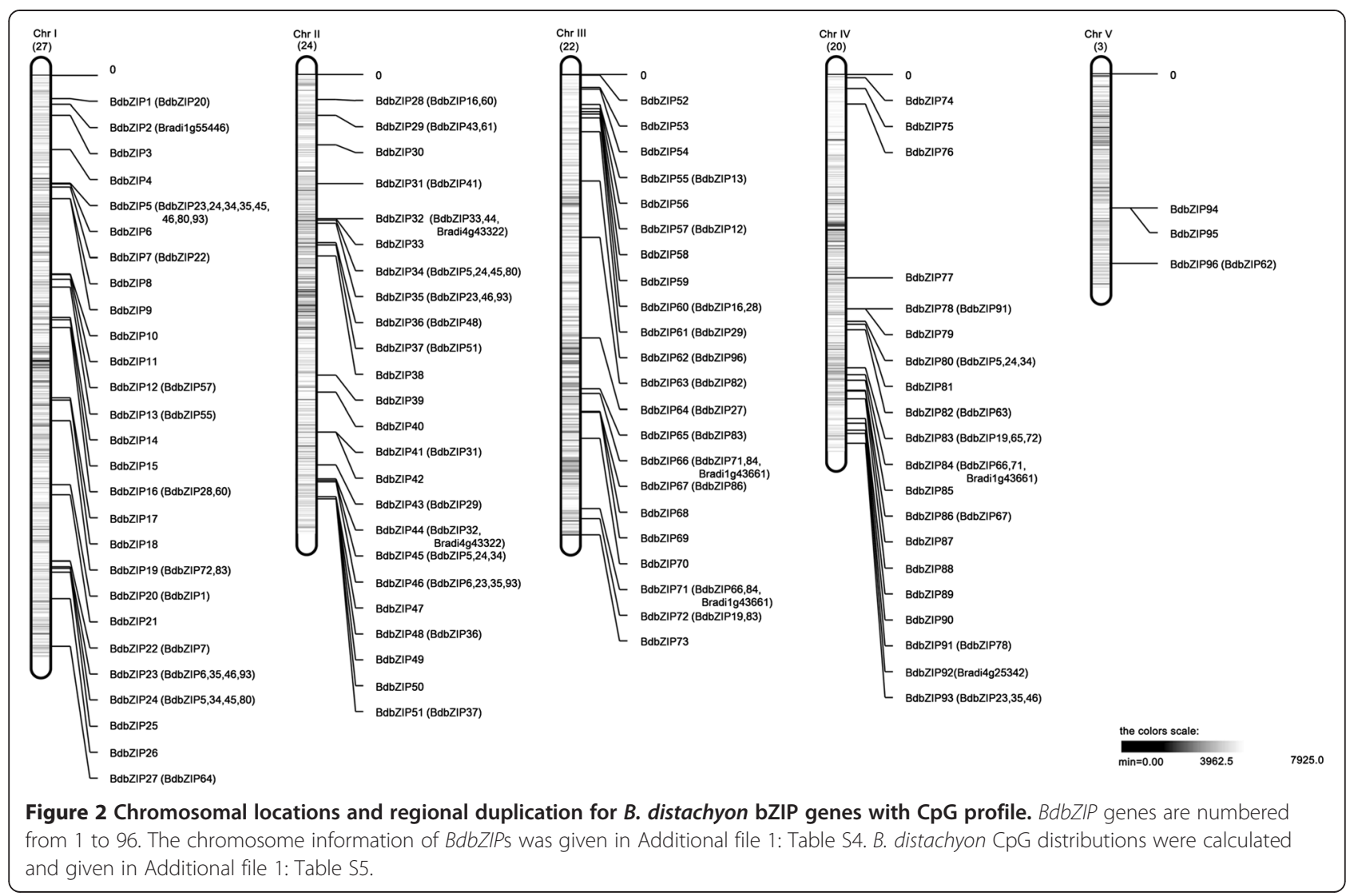

rice [10]. This result further revealed why $B$. distachyon had high amount of bZIP genes.

Much evidence showed that CpG content and distribution might have influence on variability in chromatin structure and gene distribution [54]. Based on this, we infered CpG content might affect the functional properties of $B d b Z I P$ s. To dig into the evolutionary relationships between BdbZIPs distribution and CpG content, we also counted the CpG content in the whole $B$. distachyon genome (Additional file 1: Table S5). The landscape of CpG content was generated and integrated to the genetic linkage map (Figure 2). It was observed that the distribution of $\mathrm{CpG}$ content was not uniform. Each chromosome had several relatively high CpG content regions (Figure 2). Except a few of BdbZIPs (BdbZIP5-7, $32-35,65,66,73$, and 83-87) were distributed in the regions of high $\mathrm{CpG}$ content, most of BdbZIP genes were scattered in the regions of low CpG content. This finding showed that those BdZIPs located in the regions of low CpG content might have high frequent mRNA transcripts, which required to implement their functions.

\section{Phylogenetic and molecular evolutionary analysis of BdbZIP genes}

Furthermore, to evaluate the evolutionary history of $B d b Z I P$ genes and relationships with other plant bZIP family genes, the bZIP proteins from other two model plants, Arabidopsis and rice, were performed for analyzing and comparing. A total of 268 bZIPs (Additional file 1: Table S6 and Additional file 1: Table S7) were detected and a phylogenetic tree was constructed (Figure 3). All bZIP proteins were grouped into 9 clades, designated as clade I to IX (Figure 3). It was observed that each clade had both AtbZIP and OsbZIP genes, which indicating that the evolution of bZIP genes was conservative between dicots and monocots. Most of the BdbZIP and OsbZIP genes were hierarchical clustered together in the same clades and exhibited closer relationship than those in AtbZIP genes (Figure 3). To identify the bZIP genes in terms of intragenome or cross-genome syntenic relationships among species $B$. distachyon, rice, and Arabidopsis, we also calculated the synonymous (Ks) and non-synonymous (Ka) substitution rates of bZIP genes among them [50]. We found that most of BdbZIP genes had syntenic regions in rice, but few in Arabidopsis (Additional file 1: Table S2). These results were consistent with the notion that $B$. distachyon and rice were monocots and their bZIP families might have the similar evolutionary patterns. However, it was still mentioned that some BdbZIP proteins were hierarchical clustered together with AtbZIP proteins in the same clade, the reason was that these BdbZIPs were expected to be 


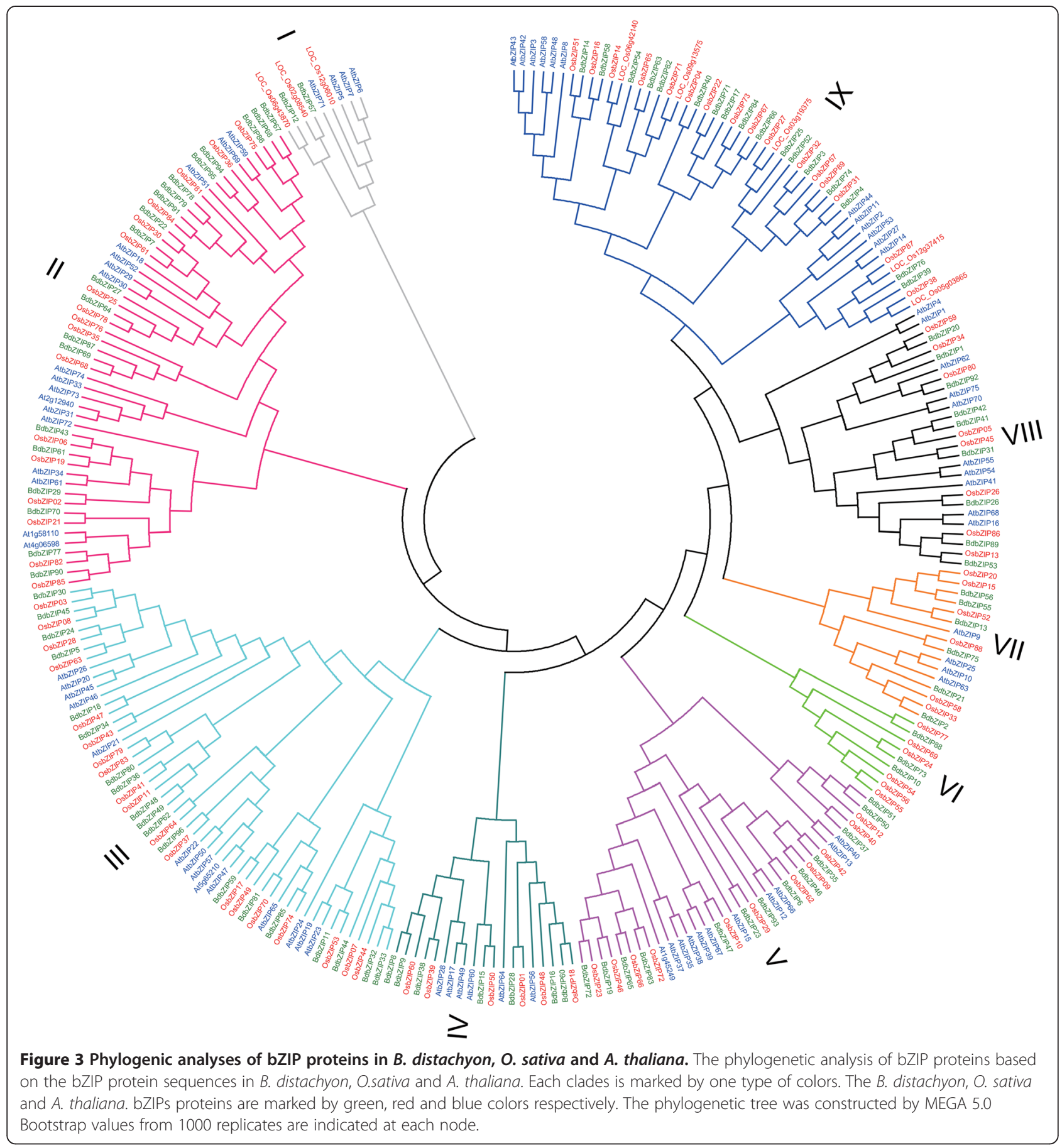

orthologs of the AtbZIP proteins and they shared other additional conserved motifs outside of bZIP domains. Furthermore, homologous bZIP genes were detected in rice and Arabidopsis using BLAST tools. Seventy-two and forty-seven BdbZIPs were found to have homologous bZIP genes in rice and Arabidopsis respectively (Additional file 1: Table S8). The comparative phylogenetic analysis and BLAST results showed that the bZIP genes in B. distachyon had closer relationships with rice than those in Arabidopsis.

\section{Gene structure analysis of $B d b Z I P$ genes}

Alternative splicing events were spread in the whole $B$. distachyon genome [55]. As the overall pattern of intron position acted as an index to the phylogenetic relationships in a gene family evolution [56], so we also examined 
the intron and exon organization of BdbZIPs (Additional file 1: Table S4). It showed that most of BdbZIPs (81 of 96 $B d b Z I P$ s) containing introns, only 15 of total BdbZIP genes were intronless. As for the genes containing introns, the numbers of introns varied from 1 to 13 . Diverse status of exon and intron splicing might be meaningful for $B d b Z I P$ gene evolution.

In addition, the intron patterns within the basic and hinge region of the bZIP domain are most conserved and particularly important for their functional evolution. Any splicing change in the hinge region would change the code of the bZIP domain of the proteins and further affect their function. Among the 81 genes containing introns, 17 had 1-2 intron/introns in this region. Based on the intron presence, position, and splicing phase, $B d b Z I P$ genes were divided into 8 patterns, which were designated as pattern a to pattern h (Figure 4 and Additional file 2: Figure S1). The pattern $\mathrm{a}, \mathrm{b}$, e, and $\mathrm{h}$ were the most prevalent. Both pattern a and pattern $b$ had one intron, pattern e had two introns and pattern $\mathrm{h}$ was intronless. But the pattern a had one intron in phase 2 (P2) in the basic region, whereas pattern b had an intron in phase 0 (P0) in the hinge region. Pattern c, d, f and $g$ were uncommon. Compared with those in rice and maize $[10,11]$, pattern $f$ and pattern $g$ were novel types. Pattern c and pattern d had one intron in P0 and P2 respectively. Pattern $\mathrm{f}$ and pattern $\mathrm{g}$ had two introns in P0. Pattern h was found in 27 BdbZIP genes, of which only 17 were intronless. The result showed that when the introns existed in the hinge region, the phase and position were conserved. However, when the introns were present in the basic regions, the positions of P0 and P2 were variable. The results showed that in $B$. distachyon, the splicing phase had remained conserved during the course of evolution of BdbZIP family genes, which were very consistent with those in rice [10]. In brief, our results showed that the intron patterns in the BdbZIP domains demonstrated more diverse than those in rice and maize.

\section{Dimerization properties of BdbZIP proteins}

The amino acids at positions nearing leucine zipper interface of leucine zipper determined the dimerization stability and specificity [13-15]. To predict the dimerization specificity and stability of all BdbZIP proteins, we adopted the standard nomenclature for the amino acid positions in heptads to arrange manually leucine zipper regions [15]. The N-terminal and C-terminal boundaries of BdbZIP leucine zippers were demarcated following the criteria used for the bZIP proteins of Arabidopsis, rice and maize $[10,11,15]$. According to their positions, the amino acids in the heptad repeats in leucine zipper region were named as position a, b, c, d, e, f, and g (Additional file 3: Figure S2). The types of amino acids present at the $g, e, a$, and $d$ positions in BdbZIP proteins were analyzed and compared with those of Arabidopsis and rice. We found that BdbZIPs had the lowest frequency of charged amino acids in these positions. This result showed that interactions between $g \leftrightarrow e$ pairs might be more prominent in regulating specificity in BdbZIPs (Figure 5A). However, the frequency of charged amino acids of BdbZIPs and OsbZIPs at $g$ and e positions had a closer proximity than that in AtbZIPs. This might due that BdbZIPs and OsbZIPs had closer evolutionary relationships. As Asns existing in a position

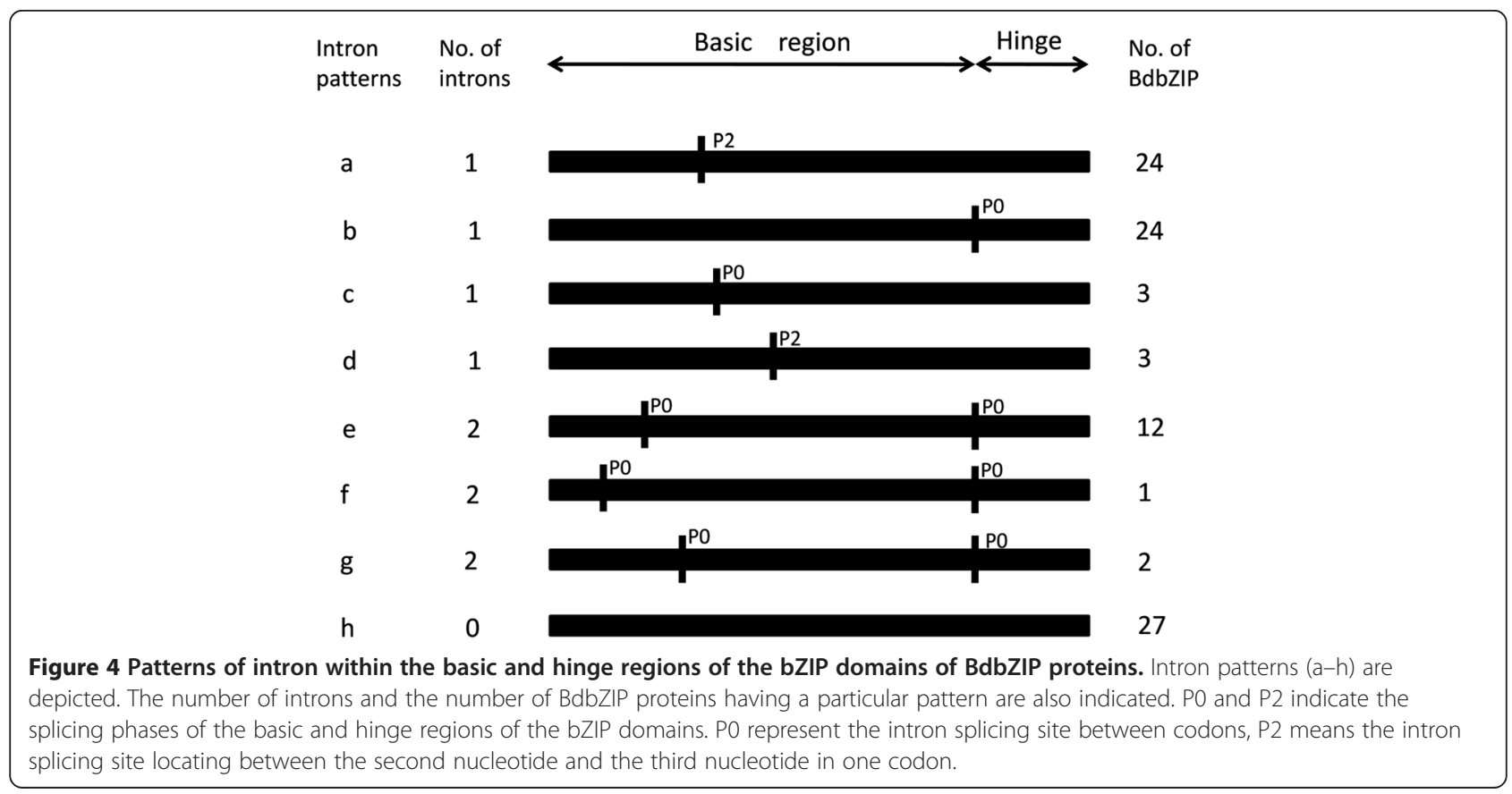



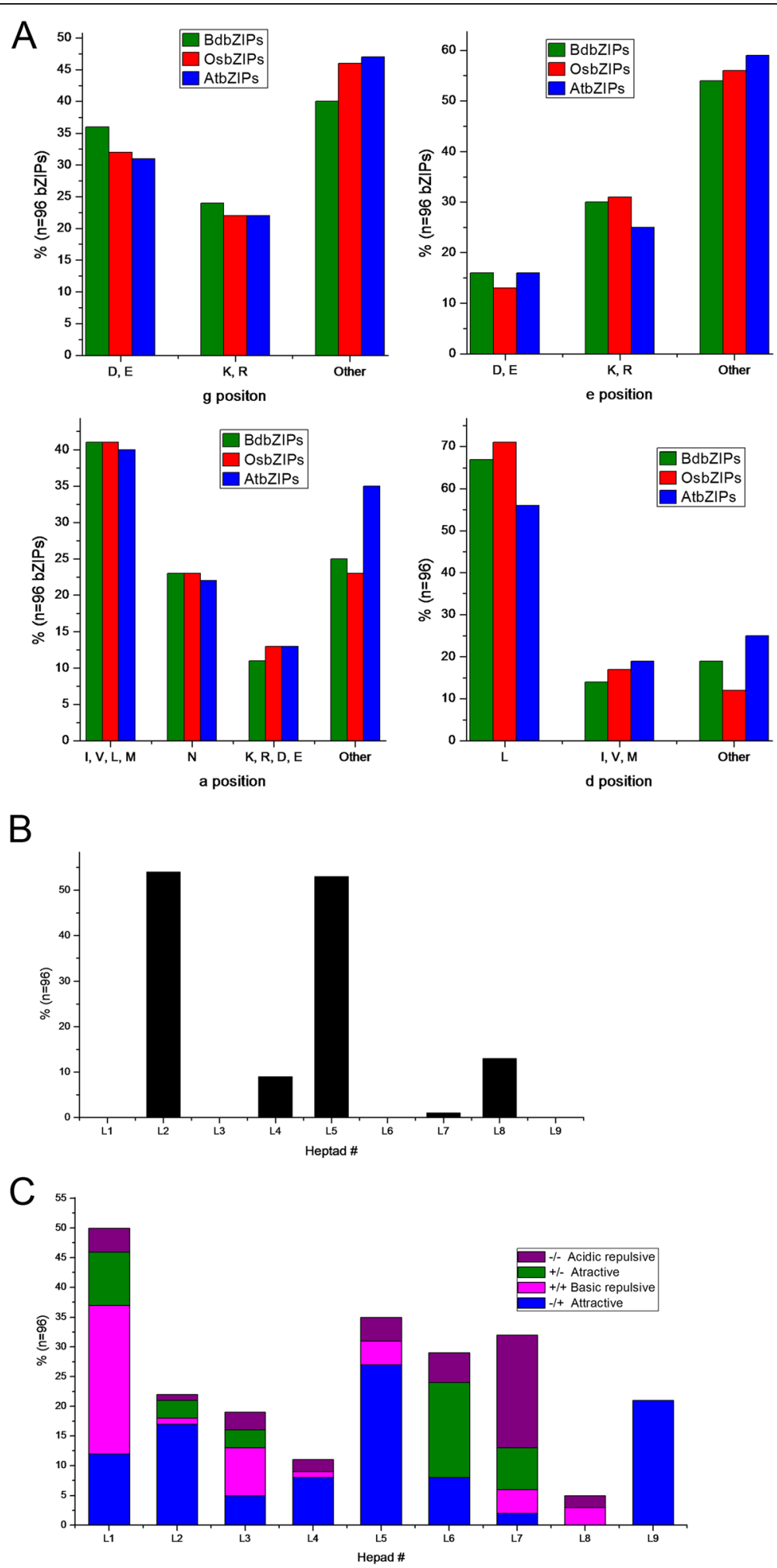

Figure 5 Amino acid analysis at the $\mathbf{g}, \mathbf{e}, \mathbf{a}$, and d positions of the leucine zippers. A. Histogram of frequency of amino acids in the g, e, a, and $d$ positions of the leucine zippers from BdbZIP, AtbZIP, and OsbZIP proteins. B. Histogram of the frequency of Asn residue in a position of the leucine zippers for all BdbZIP proteins. C. Histogram of the frequency of attractive or repulsive g↔e pairs per heptad for all BdbZIP proteins. 
contribute the most to dimerization specificity [57], we also examined the frequency of Asn in a position. About $23 \%$ of amino acids presenting at a position are Asns (Figure $5 \mathrm{~A}$ ), and about $55 \%$ of Asns in a position in heptad 2 and heptad 5 were observed in BdbZIPs (Figure 5B). The similar phenomena had been observed in AtbZIP, OsbZIPs and ZmbZIPs [10,11,15]. It was known that the leucine present in $d$ position was responsible for dimerization stability [57], so the leucine in the $d$ positions was calculated. The abundance of leucine of BdbZIPs in $\mathrm{d}$ positions was $68 \%$, which was slightly lower than that in OsbZIPs (71\%) and significantly greater than that in AtbZIPs (56\%). The number of heptads was variable and ranged from four to nine (Additional file 3: Figure S2), and higher frequency of leucine was speculated to be responsible for dimer stability of long zippers.

To evaluate the contribution of charged residues responsible for dimerization properties of BdbZIPs, the frequency of attractive and repulsive g↔e pairs in each heptad of BdbZIP leucine zippers was computed and the corresponding histogram was demonstrated (Figure 5C). It was found that the frequency of interactive $\mathrm{g} \leftrightarrow \mathrm{e}$ pairs was the maximum in the first heptad, with a sharp decrease in the next three heptads (L2, L3, and L4). Then the trend increased in the fifth heptad and decreased sharply in the eighth heptad. Moreover, only repulsive $\mathrm{g} \leftrightarrow \mathrm{e}$ pairs were observed in the eighth heptad and attractive $g \leftrightarrow \mathrm{e}$ pairs were observed in the ninth heptad. Attractive $\mathrm{g} \leftrightarrow \mathrm{e}$ pairs and presence of Asns in a position contribute to homo-dimerization. Repulsive and incomplete $\mathrm{g} \leftrightarrow \mathrm{e}$ pairs, and charged residues in a positions may favour hetero-dimerization. Based on this principle, all BdbZIPs were classified into three sub-families (subfamily I, II, and III, Additional file 3: Figure S2). According to above analyses, we observed that the dimerization patterns in the leucine zippers of BdbZIPs were more complex and diverse than those in other species. Our results indicated that there were many BdbZIPs with trends to form homo-dimerization.

\section{BdbZIP proteins structure and expression patterns}

It was important to answer the question whether BdbZIP protein structure had any correlation with their functions in different tissues/organs. Due that motifs contributed to determine specific functions for gene members, the additional conserved motifs of bZIPs had been detected and classified in plant species $[10,11,23]$. Based on sequence similarity of conserved motifs, a total of 15 conserved motifs including the bZIP domain were identified (Figure 6). According to the presence of the bZIP region and the additional conserved motifs, we classified 96 BdbZIP proteins into 5 groups (group I-V, Figure 6). All the predicated motifs were exhibited as Additional file 4: Figure S3.
Furthermore, we also investigated tissues/organs specific expression pattern of BdbZIPs genes. Four tissues/ organs including root, stem, leaf, and early spikelet were selected. The $-\triangle \Delta C T$ changes and fold changes of 96 $B d b Z I P$ genes were calculated (Figure 6 and Additional file 1: Table S9). Our results showed that the expression levels of BdbZIP genes in four tissues/organs displayed with different patterns.

BdbZIP protein structure results showed that except for motif 1 and 7, most of motifs had just one copy. Certain motifs appeared in specific groups and some motifs were shared by several groups. This phenomenon might reflect the case that the functions of some conserved motifs were important and diverse in BdbZIPs. A large number of BdbZIP genes belonged to group I and II behaved high expression levels. Except some members in group III, IV, and V had higher expression, the expression for most of the BdbZIP genes in these three groups was relatively low. It should be noted that though some of BdbZIP genes with same structure were grouped to the same groups, the expression patterns were not completely consistent with the gene structural profiles. These results indicated that the structure of BdbZIP protein was not the single factor in determining their functions in different tissues/organs.

\section{Stress expression analysis of BdbZIP genes}

Plant growth and productivity are greatly affected by various environmental stresses. Stress tolerance is an intricate phenomenon because plants may undergo multiple abiotic stresses, which are the principle cause of reducing crop yields. Given the potential roles of BdbZIP genes may play in response to environmental stresses in $B$. distachyon, it is of great importance to demonstrate all $B d b Z I P$ gene expression profiles under multiple stresses. To investigate the expression patterns, we treated $B$. distachyon with 3 major types of abiotic stresses including environmental factors (cold, heat, $\mathrm{H}_{2} \mathrm{O}_{2}$, PEG, and $\mathrm{NaCl}$ ), heavey metals $(\mathrm{Cu}, \mathrm{Zn}, \mathrm{Mn}, \mathrm{Cd}$, and $\mathrm{Pb})$ and phytohormones (SA, 6-BA, ABA, and MeJA) respectively. We obtained 4 major clusters (I, II, III, and IV) after hierarchical clustering analysis of all the data upon different types of stress treatments (Figure 7 and Additional file 1: Table S10). According to the expression profile, BdbZIPs in cluster I showed down regulation upon almost all three major treatments except ABA and MeJA treatments. As for BdbZIP genes in cluster II, we observed that they were up-regulated upon both environmental factors and phytohormone treatments, but they were down-regulated under heavy metal treatments (Figure 7). The BdbZIP genes in cluster III and cluster IV had no obvious expression patterns upon different types of stress treatments (Figure 7). 


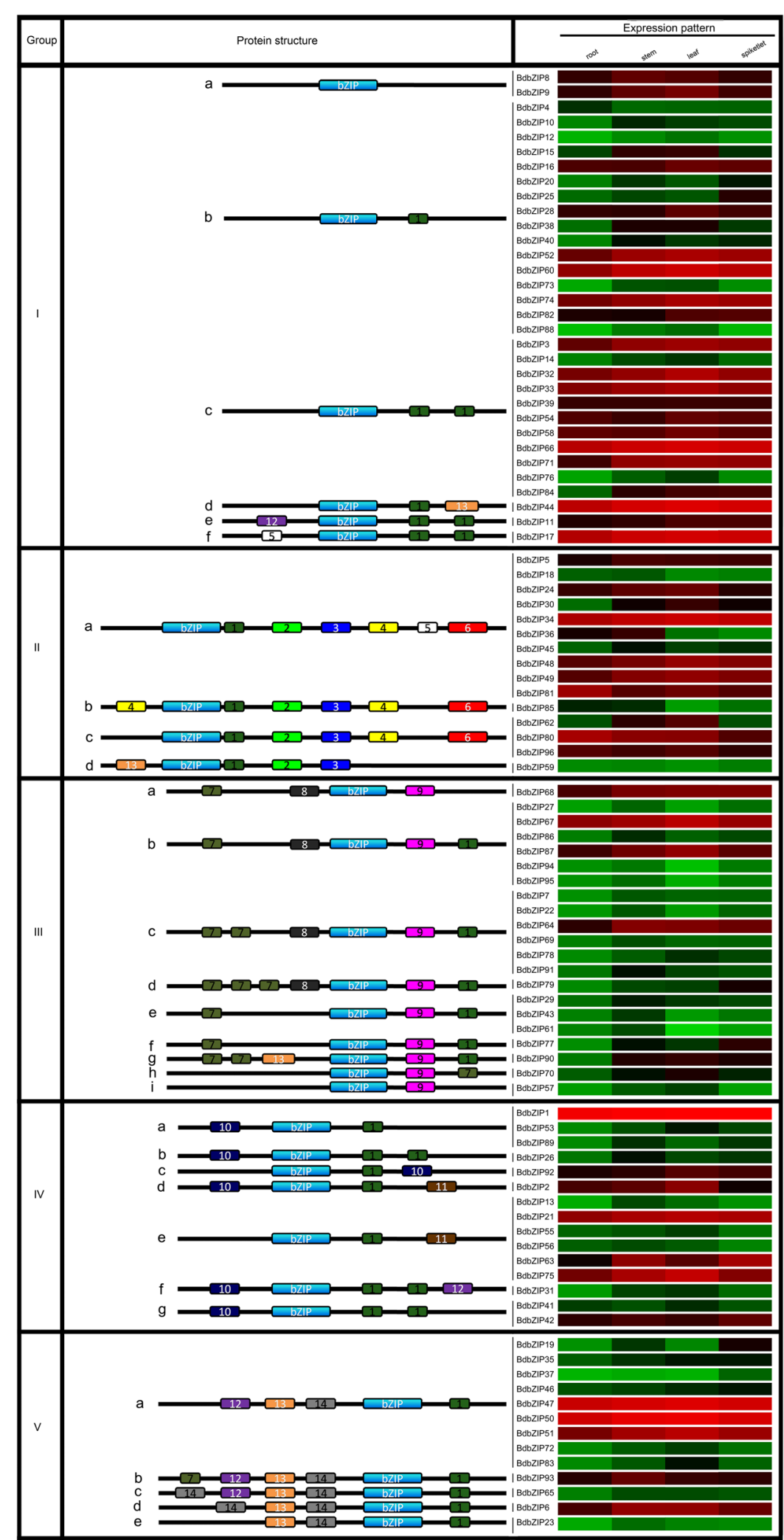

Figure 6 (See legend on next page.) 
(See figure on previous page.)

Figure 6 Tissue-specific expression profiles of BdbZIP genes with bZIP domain and motif information identified by MEME. Tissue-specific expression analysis was performed. Four tissues/organs including root, stem, leaf, and early spikelet were examined. The position of the bZIP domain and the presence of additional conserved motifs outside the bZIP domain were identified by MEME. BdbZIP domains are shown in blue. Additional motifs are marked in different color boxes with numbers 1 to 14, where the same number refers to the same motif present in the different BdbZIP proteins. The details of predicted conserved motifs are given in Additional file 2: Figure S1.

We also investigated whether the BdbZIP genes expression cluster had any enrichment with specific phylogenic clade genes, intron pattern, dimerization pattern or motif groups. It was observed that the expression of BdbZIPs in cluster II behaved heavy metal specific expression (Figure 7). We found that this cluster was composed of the high proportion of BdbZIPs belong to intron pattern b (46\%), c (67\%), d (67\%), and e (50\%). So we proposed that the 4 type of intron patterns were crucial in regulation of BdbZIPs responding to heavy metal stresses.

In order to further look BdbZIP genes expression pattern to specific group of treatments, we also did hierarchical cluster analysis of the BdbZIP genes expression according to environment factors, heavy metal stresses and phytohormone treatments respectively (Additional file 5: Figure S4, Additional file 6: Figure S5, Additional file 7: Figure S6).

\section{Environmental factors (cold, heat, $\mathrm{H} 2 \mathrm{O} 2, \mathrm{PEG}$, and $\mathrm{NaCl}$ )}

The proportion of down-regulated to up-regulated BdbZIP genes under environmental factors' treatment was approximately $50 \%$ to $50 \%$. Too high and low temperature were major negative factors on plant development due to the limiting the geographical locations suitable for plant growing and led to catastrophic loss of crop yield [58,59]. To uncover mechanism underlying temperature stresses, $B$. distachyon plants were subjected to heat and cold stress and a set of up- and down- regulated genes were identified, suggesting a prominent role for the bZIP genes responding to these environmental factors. Compared with the global analysis of the transcriptome of $B$. distachyon in cold, heat, drought and salt stress [45], 22 BdbZIPs were fell into 13 of 22 modules (Additional file 1: Table S11), which illustrated certain BdbZIPs were significant in processes of stress tolerance. Previous studies had shown that when rice was treated with cold, the bZIP genes $O s b$ ZIP14, 65, and 83 behaved down-regulated [60]. The expression patterns of these OsbZIPs were similar to their homologous BdbZIP54, 63, and 80 respectively. Moreover, many evidences had proposed that excessive $\mathrm{NaCl}$ was toxic to plants, because $\mathrm{NaCl}$ caused cellular ion imbalances and hyperosmotic stress [61]. So, we also examined the expression profiles of BdbZIPs under $\mathrm{NaCl}$ and osmotic (PEG) tolerance (Additional file 5: Figure S4 and Additional file 1: Table S10). In salt stress environment, the expressions of BdbZIP30 and BdbZIP41 were increased, which were very similar to their homologous OsbZIP63 and OsbZIP05 respectively [60].

\section{Heavy metal stresses}

With the development of industry, heavy metals contaminations are well known serious problems. Phytoremediation technologies including hyperaccumulation and uptake widely used to remove heavy metal pollutants are particularly important. Significant progresses have been made in recent years in native plants or genetic modified plants for phytoremediation of pollutants.

Genes associated with heavy metal tolerance or accumulation were identified in green alga, poplar, and maize [62-67]. To gain further insight of the potential roles of $B d b Z I P$ s may play in phytoremediation, we examined their expression patterns under heavy metals of $\mathrm{Zn}, \mathrm{Mn}$, $\mathrm{Cu}, \mathrm{Cd}$, and $\mathrm{Pb}$. A series of BdbZIPs sensitive to heavy metal were detected (Additional file 6: Figure S5). Eighty percent of the BdbZIP genes were suppressed by heavy metal treatments (Additional file 6: Figure S5). The expression patterns of the BdbZIP genes under $\mathrm{Zn}$ and $\mathrm{Mn}$ were similar (Additional file 6: Figure S5).

It was notable that after dealing with heavy metals, there existed a few of $B d b Z I P s$ with high expression levels. Those BdbZIP genes with specific expression under heavy metal treatments might be potential for application of phytoremediation.

\section{Pytohormones' treatments}

Phytohormones act as endogenous messengers when plants go through stress. During responding to environmental stresses, phytohormones such as auxin, ABA, salicylic acid, gibberellic acid play key roles and coordinate various signal transduction pathways [68]. In plant treated with exogenous hormones, the genome-wide transcript profiles changed rapidly and transiently [69]. Complex networks of transcription factors regulation by phytohormones under abiotic stresses had been reported [68]. In this part, we treated $B$. distachyon seedlings with 4 types of phytohormones (SA, 6-BA, ABA, and MeJA) and investigated the $B d b Z I P \mathrm{~s}$ expression patterns. We found about $75 \%$ of $B d b Z I P$ genes were up-regulated upon these phytohormes treatments (Additional file 7: Figure S6). The expression profile of BdbZIP genes under SA and 6-BA treatments had similar expression patterns. The expression pattern of BdbZIP genes under 


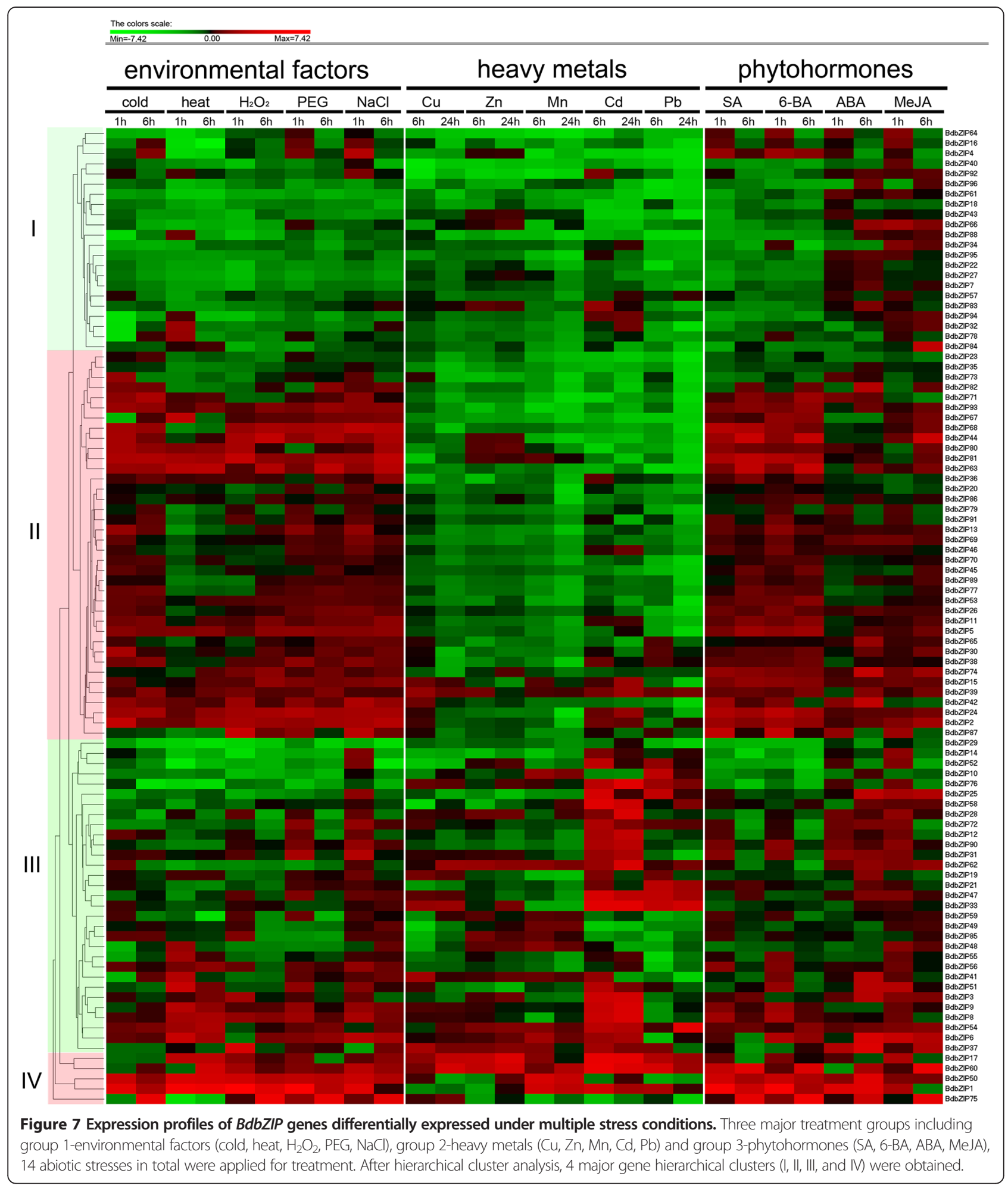

$\mathrm{ABA}$ and MeJA were similar. Our findings showed that the expression patterns of $B d b Z I P$ s can be regulated by different phytohormones. So we proposed that stress tolerance of $B$. distachyon could be adjusted by applying different phytohormones.

\section{Conclusions}

Ninety six bZIP genes were first identified from the new grass model plant B.distachon. The BdbZIP genes chromosomal localization with gene duplication and CpG density were analyzed. Phylogenic analysis of these 
genes with their counterpart species of rice and Arabidopsis were investigated. Further characterization of bZIP domain of these genes in terms of exon splicing of basic and hinge region and identification of dimerization groups were performed. Finally, genes expression profiling of all $B d b Z I P$ genes upon 14 different stress conditions in B.distachon were obtained.

Most of the BdbZIP genes were located in the regions with low CpG density in chromosomes. BdbZIP gene duplications were widespread throughout the $B$. distachyon genome. Evolutionary analyses suggested that $B$. distachyon and monocot species have the similar evolutionary patterns, which lies two points: (1) B. distachyon and other monocot species maintained the similar and high number of bZIP genes, and (2) Seventy-five percent of total BdbZIPs have homologous genes in rice. bZIP domain characterization in terms of exon splicing of basic and hinge region and dimerization patterns of leucine zipper exhibited more complex and diverse than those in O. sativa and A. thalinana. All BdbZIP domains were classified into 3 major dimerization groups, and those BdbZIPs forming homodimerization were clustered into the same expression clusters.

Multiple stresses expression profile showed that BdbZIPs exhibited significant expression patterns, and those BdbZIPs responding to heavy metal treatments showed opposite expression pattern to those of the treatments of environmental factors and phytohormones. Certain BdbZIPs with expression level of fold changes $\geq 2$ up- and downregulated upon multiple-stress treatments were screened.

Abiotic stresses are important research areas of investigating mechanisms associated with crops yields under stress conditions. Identification of novel BdbZIP genes associated with stress tolerance and development of some strategies to obtain stress-tolerant plants are our currently major topics or future researches. Determination of the up-regulated, down-regulated, or stress specific $B d b Z I P$ genes, and utilizing the $B d b Z I P$ genes to improve productivity of grass plants and cereal crops upon complicated stress environment are crucial and significant.

\section{Additional files}

Additional file 1: Table S1. Treatment conditions. Table S2. The syntenic relationships of bZIPs in $B$. distrachyon, rice, and Arabidopsis. Table S3. Primers used in this study. Table S4. Identification of BdbZIP proteins and their related information. Table S5. CpG numbers in $B$. distachyon genome. Table S6. BdbZIPs, OsbZIPs and AtbZIPs used. Table S7. BdbZIP protein sequences. Table S8. Homologous bZIPs in rice and Arabidopsis. Table S9. Tissue specific expression datas. Table S10. Stress expression datas. Table S11. BdbZIPs in modules.

Additional file 2: Figure S1. Intron patterns within the basic and hinge regions of $B$. distachyon bZIP domains.

Additional file 3: Figure S2. The leucine zipper regions. BdbZIPs were classified as three major sub-families according to the predicted dimerization specificity. BdbZIPs in Sub-family I are homo-dimerization specific. BdbZIPs in Sub-family II are homo-hetero-dimerization, and BdbZIPs in sub-family III are hetero-dimerization specific. The leucine zipper regions are divided into heptads (gabcdef) from L0 to L9 to display the potential $g \leftrightarrow e^{\prime}$ pairs. Based on the electrostatic charges at the $g$ and $e$ positions, the $\mathrm{g} \leftrightarrow$ e pairs were grouped into 4 types, which were displayed with 4 different colors: the frequency of the attractive basic-acidic pairs $(+/-)$ was displayed with green color, attractive acidic-basic pairs (-/+) was displayed with blue color, repulsive acidic pairs (-/-) was displayed with purple color, and repulsive basic pairs $(+/+)$ was displayed with pink color. If single amino acid at the positions e or $\mathrm{g}$ is charged, the residue is colored pink for basic amino acid and purple for acidic amino acid. If a or $d$ position is polar, it is colored grey and if either is charged, it is colored orange. Asparagines at a position are colored red. The prolines and glycines are boxed to indicate a potential break in a-helix. The predicted C-terminal boundary is denoted by the symbol $\bullet$

Additional file 4: Figure S3. The additional conserved motifs of BdbZIP proteins predicted by MEME.

Additional file 5: Figure S4. Exression profiles of BdbZIP genes differentially expressed under environmental factors (cold, heat, $\mathrm{H}_{2} \mathrm{O}_{2}$, PEG, $\mathrm{NaCl}$ ).

Additional file 6: Figure S5. Exression profiles of BdbZIP genes differentially expressed under heavy metal factors (Cu, Zn, Mn, Cd, Pb).

Additional file 7: Figure S6. Exression profiles of BdbZIP genes differentially expressed under hytohormones (SA, 6-BA, ABA, MeJA).

\section{Competing interests}

The authors declare that they have no competing interests.

\section{Authors' contributions}

$X \mathrm{~L}$ and $\mathrm{ZC}$ designed the experiment. $\mathrm{XL}$ conducted the experiment and the data analysis, $\mathrm{XL}$ and ZC wrote this manuscript. All authors read and approved the final manuscript.

\section{Acknowledgements}

This work was supported by grant for Starting Package to the Research Group of Plant Abiotic Stress and Plant Genome Evolution in Shanghai Chenshan Plant Science Research Centre, Chinese Academy of Sciences, and Shanghai Chenshan Botanic Garden from Shanghai Landscaping Administrative Bureau (NO. F0112423, F0122415, F122423, F132426).

Received: 15 August 2014 Accepted: 9 March 2015

Published online: 22 March 2015

\section{References}

1. Liu JX, Srivastava R, Howell SH. Stress-induced expression of an activated form of AtbZIP17 provides protection from salt stress in Arabidopsis. Plant Cell Environ. 2008;31:1735-43.

2. Schlogl PS, Nogueira FT, Drummond R, Felix JM, De Rosa Jr VE, Vicentini R, et al. Identification of new ABA- and MEJA-activated sugarcane bZIP genes by data mining in the SUCEST database. Plant Cell Rep. 2008;27:335-45.

3. Yamamoto MP, Onodera Y, Touno SM, Takaiwa F. Synergism between RPBF Dof and RISBZ1 bZIP activators in the regulation of rice seed expression genes. Plant Physiol. 2006;141:1694-707.

4. Yang O, Popova OV, Suthoff U, Luking I, Dietz KJ, Golldack D. The Arabidopsis basic leucine zipper transcription factor AtbZIP24 regulates complex transcriptional networks involved in abiotic stress resistance. Gene. 2009:436:45-55.

5. Yang YG, Lv WT, Li MJ, Wang B, Sun DM, Deng X. Maize membrane-bound transcription factor Zmbzip17 Is a key regulator in the crosstalk of ER quality control and ABA signaling. Plant Cell Physiol. 2013;54:2020-33.

6. Hurst HC. Transcription factors. 1: bZIP proteins. Protein Profile. 1994;1:123-68,

7. Ellenberger TE, Brandl CJ, Struhl K, Harrison SC. The GCN4 basic region leucine zipper binds DNA as a dimer of uninterrupted alpha helices: crystal structure of the protein-DNA complex. Cell. 1992;71:1223-37.

8. Vinson CR, Sigler PB, McKnight SL. Scissors-grip model for DNA recognition by a family of leucine zipper proteins. Science. 1989;246:911-6.

9. Fassler J, Landsman D, Acharya A, Moll JR, Bonovich M, Vinson C. B-ZIP proteins encoded by the Drosophila genome: evaluation of potential dimerization partners. Genome Res. 2002;12:1190-200. 
10. Nijhawan A, Jain M, Tyagi AK, Khurana JP. Genomic survey and gene expression analysis of the basic leucine zipper transcription factor family in rice. Plant Physiol. 2008;146:333-50.

11. Wei KF, Chen J, Wang YM, Chen YH, Chen SX, Lin YN, et al. Genome-wide analysis of bZIP-encoding genes in maize. DNA Res. 2012;19:463-76.

12. Fong $\mathrm{JH}$, Keating $A E$, Singh $M$. Predicting specificity in bZIP coiled-coil protein interactions. Genome Biol. 2004;5:RII.

13. Newman JR, Keating AE. Comprehensive identification of human bZIP interactions with coiled-coil arrays. Science. 2003;300:2097-101.

14. Vinson C, Myakishev M, Acharya A, Mir AA, Moll JR, Bonovich M Classification of human B-ZIP proteins based on dimerization properties. Mol Cell Biol. 2002;22:6321-35.

15. Deppmann CD, Acharya A, Rishi V, Wobbes B, Smeekens S, Taparowsky EJ, et al. Dimerization specificity of all 67 B-ZIP motifs in Arabidopsis thaliana: a comparison to homo sapiens B-ZIP motifs. Nucleic Acids Res. 2004;32:3435-45.

16. Nardmann J, Werr W. The evolution of plant regulatory networks: what Arabidopsis cannot say for itself. Curr Opinion Plant Biol. 2007;10:653-9.

17. Xu F, Park MR, Kitazumi A, Herath V, Mohanty B, Yun SJ, et al. Cis- regulatory signatures of orthologous stress-associated bZIP transcription factors from rice, sorghum and Arabidopsis based on phylogenetic footprints. BMC Genomics. 2012;13:497-502.

18. Correa LGG, Riano-Pachon DM, Schrago CG, dos Santos RV, Mueller-Roeber B, Vincentz M. The role of bZIP transcription factors in green plant evolution: adaptive features emerging from four founder. PLoS One. 2008;3:e2944.

19. Mahajan S, Tuteja N. Cold, salinity and drought stresses: an overview. Arch Biochem Biophys. 2005;444:139-58.

20. Xiong L, Schumaker KS, Zhu JK. Cell signaling during cold, drought, and salt stress. Plant Cell. 2002;14 (Suppl):S165-83.

21. Urano K, Kurihara Y, Seki M, Shinozaki K. 'Omics' analyses of regulatory networks in plant abiotic stress responses. Curr Opin Plant Biol. 2010;13:132-8.

22. Zhang $H$, Jin JP, Tang L, Zhao Y, Gu XC, Gao G, et al. PlantTFDB 2.0: update and improvement of the comprehensive plant transcription factor database. Nucleic Acids Res. 2011;39(Database issue):1114-7.

23. Jakoby M, Weisshaar B, Droge-Laser W, Vicente-Carbajosa J, Tiedemann J, Kroj T, et al. bZIP transcription factors in Arabidopsis. Trends Plant Sci. 2002;7:106-11.

24. Baloglu MC, Eldem V, Hajyzadeh M, Unver T. Genome-wide analysis of the bZIP transcription factors in cucumber. PLoS One. 2014;9:e96014.

25. Wang JZ, Zhou JX, Zhang BL, Vanitha J, Ramachandran S, Jiang SY. Genome-wide expansion and expression divergence of the basic leucine zipper transcription factors in higher plants with an emphasis on sorghum. J Integr Plant Biol. 2011;53:212-31.

26. Liu JY, Chen NN, Chen F, Cai B, Santo SD, Tornielli GB, et al. Genome-wide analysis and expression profile of bZIP transcription factor gene family in grapevine (Vitis vinifera). BMC Genomics. 2014;15:281-99.

27. Liu CT, Mao BG, Ou SJ, Wang W, Liu LC, Wu YB, et al. OsbZIP71, a bZIP transcription factor, confers salinity and drought tolerance in rice. Plant $\mathrm{Mol}$ Biol. 2014;84:19-36.

28. Tang $\mathrm{N}$, Zhang $\mathrm{H}$, Li XH, Xiao $\mathrm{JH}$, Xiong LZ. Constitutive activation of transcription factor OsbZIP46 improves drought tolerance in rice. Plant Physiol. 2012;158:1755-68.

29. Liu CT, Wu YB, Wang XP. bZIP transcription factor OsbZIP52/RISBZ5: a potential negative regulator of cold and drought stress response in rice. Planta. 2012;235:1157-69.

30. Finkelstein RR, Lynch TJ. The Arabidopsis abscisic acid response gene ABI5 encodes a basic leucine zipper transcription factor. Plant Cell. 2000:12:599-609.

31. Ji XY, Liu GF, Liu YJ, Zheng $L$, Nie XG, Wang YG. The bZIP protein from Tamarix hispida, ThbZIP1, is ACGT elements binding factor that enhances abiotic stress signaling in transgenic Arabidopsis. BMC Plant Biol. 2013;13:151-64.

32. He S, Shan W, Kuang JF, Xie H, Xiao YY, Lu WJ, et al. Molecular characterization of a stress-response bZIP transcription factor in banana. Plant Cell Tiss Org Cult. 2013;113:173-87.

33. Al-Najar H, Kaschl A, Schulz R, Romheld V. Effect of thallium fractions in the soil and pollution origins on TI uptake by hyperaccumulator plants: a key factor for the assessment of phytoextraction. Int J Phytoremediation. 2005;7:55-67.
34. Anderson L, Walsh MM. Arsenic uptake by common marsh fern Thelypteris palustris and its potential for phytoremediation. Sci Total Environ. 2007;379:263-5.

35. Chandra R, Yadav S. Phytoremediation of $\mathrm{Cd}, \mathrm{Cr}, \mathrm{Cu}, \mathrm{Mn}, \mathrm{Fe}, \mathrm{Ni}, \mathrm{Pb}$ and $\mathrm{Zn}$ from aqueous solution using Phragmites cummunis, Typha angustifolia and Cyperus esculentus. Int J Phytoremediation. 2011;13:580-91.

36. Gupta DK, Huang HG, Corpas FJ. Lead tolerance in plants: strategies for phytoremediation. Environ Sci Pollut Res Int. 2013;20:2150-61.

37. Hussein HS, Ruiz ON, Terry N, Daniell H. Phytoremediation of mercury and organomercurials in chloroplast transgenic plants: enhanced root uptake, translocation to shoots, and volatilization. Environ Sci Technol. 2007:41:8439-46.

38. Jacob DL, Borchardt JD, Navaratnam L, Otte ML, Bezbaruah AN. Uptake and translocation of Ti from nanoparticles in crops and wetland plants. Int J Phytoremed. 2013;15:142-53.

39. Pineau C, Loubet S, Lefoulon C, Chalies C, Fizames C, Lacombe B, et al. Natural variation at the FRD3 MATE transporter locus reveals cross-talk between Fe homeostasis and Zn tolerance in Arabidopsis thaliana. PLoS Genet. 2012;8:e1003120.

40. International Brachypodium I. Genome sequencing and analysis of the model grass Brachypodium distachyon. Nature. 2010;463:763-8.

41. Alves SC, Worland B, Thole V, Snape JW, Bevan MW, Vain P. A protocol for Agrobacterium-mediated transformation of Brachypodium distachyon community standard line Bd21. Nat Protoc. 2009;4:638-49.

42. Trabucco GM, Matos DA, Lee SJ, Saathoff AJ, Priest HD, Mockler TC, et al. Functional characterization of cinnamyl alcohol dehydrogenase and caffeic acid O-methyltransferase in Brachypodium distachyon. BMC Biotechnol. 2013:13:61-80.

43. Trafford K, Haleux P, Henderson M, Parker M, Shirley NJ, Tucker MR, et al. Grain development in Brachypodium and other grasses: possible interactions between cell expansion, starch deposition, and cell-wall synthesis. J Exp Bot. 2013;64:5033-47.

44. Verelst W, Bertolini E, De Bodt S, Vandepoele K, Demeulenaere M, Pe ME, et al. Molecular and physiological analysis of growth-limiting drought stress in Brachypodium distachyon leaves. Mol Plant. 2013;6:311-22.

45. Priest HD, Fox SE, Rowley ER, Murray JR, Michael TP, Mockler TC. Analysis of global gene expression in Brachypodium distachyon reveals extensive network plasticity in response to abiotic stress. PLoS One. 2014;9:e87499.

46. Reinke AW, Baek J, Ashenberg O, Keating AE. Networks of bZIP protein-protein interactions diversified over a billion years of evolution. Science. 2013:340:730-4.

47. Jin JP, Zhang H, Kong L, Gao G, Luo JC. PlantTFDB 3.0: a portal for the functional and evolutionary study of plant transcription factors. Nucleic Acids Res. 2013:42(Database issue):1182-7.

48. Tamura K, Peterson D, Peterson N, Stecher G, Nei M, Kumar S. MEGA5: molecular evolutionary genetics analysis using maximum likelihood, evolutionary distance, and maximum parsimony methods. Mol Biol Evol. 2011;28:2731-9.

49. Lee $T H$, Tang HB, Wang XY, Paterson AH. PGDD: a database of gene and genome duplication in plants. Nucleic Acids Res. 2013. doi:10.1093/nar/ gks1104.

50. Liu RH, Meng JL. MapDraw: a microsoft excel macro for drawing genetic linkage maps based on given genetic linkage data. Hereditas 2003:25:317-21

51. Liu X, Zuo KJ, Xu JT, Li Y, Zhang F, Yao HY, et al. Functional analysis of GbAGL1, a D-lineage gene from cotton (Gossypium barbadense). J Exp Bot. 2010:61:1193-203.

52. Cannon SB, Mitra A, Baumgarten A, Young ND, May G. The roles of segmental and tandem gene duplication in the evolution of large gene families in Arabidopsis thaliana. BMC Plant Biol. 2004;4:10.

53. Paterson AH, Bowers JE, Chapman BA. Ancient polyploidization predating divergence of the cereals, and its consequences for comparative genomics. Proc Natl Acad Sci U S A. 2004:101:9903-8.

54. Arndt PF, Hwa T, Petrov DA. Substantial regional variation in substitution rates in the human genome: importance of GC content, gene density, and telomere-specific effects. J Mol Evol. 2005;60:748-63.

55. Walters B, Lum G, Sablok G, Min XJ. Genome-wide landscape of alternative splicing events in Brachypodium distachyon. DNA Res. 2013;20:163-71.

56. Patthy L. Intron-dependent evolution: preferred types of exons and introns. FEBS Lett. 1987:214:1-7. 
57. Acharya A, Ruvinov SB, Gal J, Moll JR, Vinson C. A heterodimerizing leucine zipper coiled coil system for examining the specificity of a position interactions: amino acids I, V, L, N, A, and K. Biochemistry. 2002;41:14122-31.

58. Bita CE, Gerats T. Plant tolerance to high temperature in a changing environment: scientific fundamentals and production of heat stress-tolerant crops. Front Plant Sci. 2013:4:273.

59. Thomashow MF. Plant cold acclimation: freezing tolerance genes and regulatory mechanisms. Annu Rev Plant Physiol Plant Mol Biol. 1999;50:571-99.

60. Wang D, Pan YJ, Zhao XQ, Zhu LH, FU BY, Li ZK. Genome-wide temporal-spatial gene expression profiling of drought responsiveness in rice. BMC Genomics. 2011;12:149.

61. Zhu JK. Plant salt tolerance. Trends Plant Sci. 2001;6:66-71.

62. Ding D, Li W, Song G, Qi H, Liu J, Tang J. Identification of QTLs for arsenic accumulation in maize. (Zea mays L.) using a RIL population. PLoS One. 2011;6:e25646.

63. Kieffer P, Dommes J, Hoffmann L, Hausman JF, Renaut J. Quantitative changes in protein expression of cadmium-exposed poplar plants. Proteomics. 2008;8:2514-30.

64. Mendoza-Cozatl DG, Jobe TO, Hauser F, Schroeder Jl. Long-distance transport, vacuolar sequestration, tolerance, and transcriptional responses induced by cadmium and arsenic. Curr Opin Plant Biol. 2011;14:554-62.

65. Quaggiotti S, Barcaccia G, Schiavon M, Nicole S, Galla G, Rossignolo V, et al. Phytoremediation of chromium using Salix species: cloning ESTs and candidate genes involved in the $\mathrm{Cr}$ response. Gene. 2007:402:68-80.

66. Rubinelli P, Siripornadulsil S, Gao-Rubinelli F, Sayre RT. Cadmium- and iron-stress-inducible gene expression in the green alga Chlamydomonas reinhardtii: evidence for $\mathrm{H} 43$ protein function in iron assimilation. Planta. 2002;215:1-13.

67. Shen YO, Zhang YZ, Chen J, Lin HJ, Zhao MJ, Peng HW, et al. Genome expression profile analysis reveals important transcripts in maize roots responding to the stress of heavy metal Pb. Physiol Plant. 2013;147:270-82.

68. Wolters $\mathrm{H}$, Jurgens $\mathrm{G}$. Survival of the flexible: hormonal growth control and adaptation in plant development. Nat Rev Genet. 2009;10:305-17.

69. Chapman EJ, Estelle M. Mechanism of auxin-regulated gene expression in plants. Annu Rev Genet. 2009;43:265-85.

\section{Submit your next manuscript to BioMed Central and take full advantage of:}

- Convenient online submission

- Thorough peer review

- No space constraints or color figure charges

- Immediate publication on acceptance

- Inclusion in PubMed, CAS, Scopus and Google Scholar

- Research which is freely available for redistribution 